\title{
El impacto en las aves por el turismo de naturaleza: una mini revisión
}

\section{Impact on birds by nature tourism: a mini-review}

\section{Hugo Antonio Curiel-Durán ${ }^{1}$ Carlos Alberto Lara-Rodríguez ${ }^{2 *}$, Citlalli Castillo-Guevara ${ }^{2}$,} Raúl Ortiz-Pulido ${ }^{3}$

${ }^{1}$ Maestría en Biotecnología y Manejo de Recursos Naturales, Centro de Investigaciones en Ciencias Biológicas, Universidad Autónoma de Tlaxcala, km 10.5 Autopista TlaxcalaSan Martín Texmelucan, San Felipe Ixtacuixtla, Tlaxcala 90120, México.

${ }^{2}$ Centro de Investigación de Ciencias Biológicas, Universidad Autónoma de Tlaxcala, km 10.5 Autopista San Martín Texmelucan- Tlaxcala, 90120 Ixtacuixtla, Tlaxcala, México

3 Centro de Investigaciones Biológicas, Instituto de Ciencias Básicas e Ingeniería, Universidad Autónoma del Estado de Hidalgo. Km 4.5 Carretera Pachuca-Tulancingo, Mineral de la Reforma, Pachuca, Hidalgo, 42184, México. *Email: laracar@posgradosuatx.com.mx https://doi.org/10.29267/mxjb.2017.2.1.37

\begin{abstract}
Birds are important part of the great diversity that exists in ecosystems with a certain degree of conservation, which may or may not be integrated into a protection or exploitation regime. The birds are a component of the landscape and the biodiversity that exists in natural areas, mainly wild species that have adapted to the disturbances, caused by the change of land use for urbanization and industrial activities, such as mass tourism that has not respected the native vegetation and the species that has in that place its habitat are displaced to places without the disturbance However that the effects on birds not only affect the behavior but also the physiological aspects of birds such as reproductive success and hormonal stress and more, this is a review about the impacts of nature based tourism on the wild birds.
\end{abstract}

Keywords: birds, tourism, impact, disturb. 


\section{RESUMEN}

Las aves son parte importante de la gran diversidad que albergan en los ecosistemas alrededor del mundo, particularmente aquellos con cierto grado de conservación, que pueden estar o no integradas a un régimen de protección o aprovechamiento. Este grupo es un componente del paisaje y de la biodiversidad que existe en áreas naturales principalmente, especies silvestres que se han adaptado a los disturbios como el cambio de uso de suelo, para la urbanización y actividades industriales, tales como el turismo de masas. Estas últimas actividades generalmente no han respetado la vegetación nativa y las especies que tiene en ese lugar su hábitat son desplazadas hacia áreas no perturbadas o en su defecto desaparecen. Estudios en las últimas dos décadas han demostrado que esta actividad turística tiene importantes efectos en las aves, particularmente en su comportamiento, fisiología, que denotan un elevado estrés ambiental y hormonal, los cuales potencialmente afectan los aspectos fisiológicos que generan estrés hormonal y afectan su éxito reproductivo.

Palabras clave: aves, diversidad, turismo, disturbio.

\section{INTRODUCCION}

Durante décadas el turismo ha experimentado un continuo crecimiento y diversificación siendo uno de los sectores económicos de mayor desarrollo en el mundo (OMT, 2005). Esta actividad genera una gran derrama económica, trascendental en el desarrollo social y económico de todo el mundo, principalmente por la generación de empleos, y la demanda de servicios las cuales implican un gasto por parte de los visitantes. (García, 2015). El turismo se clasifica de acuerdo al componente espacial, temporal o incluso al propósito del viaje o estancia sin motivo de lucro (como visitante) (Ibáñez, 2007).

\subsection{Tipologías del turismo}

El sistema turístico está integrado por la oferta, la demanda, el espacio geográfico y los operadores de mercado (Balladares, 2015). En la actividad turística existe dos grandes modalidades: 1) turismo tradicional de hoteles y resorts y 2) el turismo alternativo. Sin embargo otra modalidad se relaciona con el tipo de actividad que realiza el turista (Ojeda, 2015; Tabla 1). 
Tabla 1. Tipos de Turismo (modificado de Bello, 2014).

\begin{tabular}{|c|c|c|c|}
\hline Tipo & Definición & Subproductos & Subsegmentos \\
\hline $\begin{array}{l}\text { Turismo de } \\
\text { Salud }\end{array}$ & $\begin{array}{l}\text { Es el desplazamiento de } \\
\text { las personas que buscan } \\
\text { mejorar su estilo } \\
\text { personal de vida hacia } \\
\text { una vida más saludable: } \\
\text { aprendiendo. }\end{array}$ & SPA's & $\begin{array}{l}\text { Técnicas } \\
\text { gimnasticas } \\
\text { especializadas } \\
\text { Tratamientos } \\
\text { especializados } \\
\text { O2 } \\
\text { SPA Resort } \\
\end{array}$ \\
\hline $\begin{array}{l}\text { Turismo de } \\
\text { Naturaleza }\end{array}$ & $\begin{array}{l}\text { Viajes en donde se busca } \\
\text { el contacto con la } \\
\text { naturaleza }\end{array}$ & $\begin{array}{l}\text { Turismo de aventura } \\
\text { Turismo Rural } \\
\text { Ecoturismo }\end{array}$ & $\begin{array}{l}\text { Turismo } \\
\text { Solidario } \\
\text { Turismo } \\
\text { experiencial } \\
\text { Ecoparks } \\
\end{array}$ \\
\hline $\begin{array}{l}\text { Turismo de } \\
\text { Reuniones }\end{array}$ & $\begin{array}{l}\text { Son viajes vinculados } \\
\text { con las actividades } \\
\text { laborales, para reuniones } \\
\text { de negocios, propósitos y } \\
\text { magnitudes. }\end{array}$ & Viajes & Convenciones \\
\hline $\begin{array}{l}\text { Turismo } \\
\text { cultural }\end{array}$ & $\begin{array}{l}\text { Es un viaje turístico } \\
\text { motivado para conocer, } \\
\text { comprender y disfrutar el } \\
\text { conjunto de rasgos o } \\
\text { lugares distintivos: }\end{array}$ & $\begin{array}{lr}\text { Museos, } & \text { sitios } \\
\text { patrimonio, } & \text { zonas } \\
\text { arqueológicas. } & \end{array}$ & $\begin{array}{l}\text { Turismo } \\
\text { religioso } \\
\text { Turismo } \\
\text { Idiomático } \\
\text { Turismo } \\
\text { Gastronómico } \\
\end{array}$ \\
\hline $\begin{array}{l}\text { Turismo } \\
\text { náutico } \\
\text { deportivo }\end{array}$ & $\begin{array}{l}\text { Turismo orientado a } \\
\text { acciones en contacto con } \\
\text { el agua, actividades } \\
\text { deportivas, dependen en } \\
\text { algunos casos de una } \\
\text { embarcación. }\end{array}$ & $\begin{array}{l}\text { Pesca deportiva } \\
\text { Golf } \\
\text { Cinegético } \\
\text { Buceo }\end{array}$ & $\begin{array}{l}\text { Triatlón } \\
\text { Maratones } \\
\text { Carreras } \\
\text { Natación }\end{array}$ \\
\hline
\end{tabular}




\section{IMPACTOS DEL TURISMO DE NATURALEZA}

El turismo de naturaleza es la actividad basada en el contacto directo con los recursos naturales, en la oferta de espacios naturales y donde la naturaleza misma es la base de la actividad turística (Ojeda, 2015). Aunque se asume que el turismo de naturaleza provee a las comunidades receptoras el medio para una mejora en la calidad de vida buscando incentivar un desarrollo sustentable, en la práctica se ha comprobado que si esto no se lleva a cabo, se tienen consecuencias negativas en el medio ambiente (Ibáñez, 2007). En este sentido una opción, que ha venido ganando adeptos alrededor del mundo, es el denominado turismo alternativo (García et al., 2015), el cual generalmente se sitúa o desarrolla en áreas protegidas naturales (las cuales cuenta con algún nivel de protección por iniciativa privada, por el estado o comunal). Este tipo de turismo basado en la naturaleza, presupone que los organizadores están conscientes de los valores naturales, presupone que los organizadores están conscientes de los valores naturales, sociales y comunitarios, y que los turistas desean interactuar con la población local. Sin embargo, a la fecha poco se conoce sobre los impactos de este tipo de actividad sobre los ambiente donde se desarrolla.

Los disturbios antropogénicos en el ambiente son variados y difieren en duración, intensidad y periodicidad (Steven, 2011). Existen esfuerzos para evaluar el turismo de naturaleza, con base en un modelo de desempeño ambiental OCDE (1993), donde se utilizan indicadores e índices aplicados a la actividad turística. En este sentido se han identificado indicadores desde el nivel municipal, estatal, regional o puntual muy importantes para la realización de diagnósticos del turismo en alguna área en particular. Por ejemplo los índices de impacto para el ambiente son en particular: calidad del agua, incendios, e índice de naturalidad (Bello, 2014).En este último se sabe que las actividades turísticas pueden tener un efecto directo sobre la fauna silvestre, particularmente porque pueden afectar sus patrones de actividad, en específico aquellos asociados al uso de recursos ya sea de alimentación, descanso o reproducción (Gill, 2007). Aunque el turismo de naturaleza depende de los ingresos económicos, las condiciones del mercado ponen en riesgo esta actividad. Sin embargo se ha demostrado que los ingresos obtenidos contribuyen significativamente a la conservación de aves amenazadas (Steven et al., 2013).

\subsection{Impactos del turismo al hábitat}

El turismo es una actividad muy importante para el desarrollo económico de muchos países, pero ya sea tradicional o alternativo existen implicaciónes en el hábitat donde se lleva a cabo. Por ejemplo el desarrollo turístico inmobiliario (construcción de hoteles, marinas, condominios, campos de golf etc.) ocasiona fuertes presiones ambientales en los ecosistemas, principalmente por la falta de planeación (PROFEPA, 2016), delitos ambientales, el incumplimiento de la normatividad por parte de los responsables sin los estudios pertinentes y, autorizaciones, ha generado impactos ambientales directamente al hábitat, y el cambio de suelo forestal. Estas actividades, afectan a la flora y fauna con el agotamiento de recursos, generando residuos peligrosos, la contaminación y obstrucción de cuerpos de agua, introducción de especies exóticas, emisión de ruidos, vibraciones por maquinaria pesada y el cambio climático (PNUMA, 2005). A este respecto, se calcula que 
las emisiones de dióxido de carbono generadas por las actividades relacionadas con la industria turística (transporte, hotelería, etc.) representan alrededor de un 4 a un $6 \%$ de las emisiones mundiales totales (OMT et al., 2007).

En ecosistemas terrestres y costeros existe un cambio de uso de suelo para el desarrollo turístico tradicional. Sin embargo el turismo con base en naturaleza se ha dirigido con base a propuestas en el uso de los recursos naturales a un bajo impacto, desde el uso de ecotecnias (por ejemplo el baño seco que ha logrado mitigar el impacto por la generación de residuos). El turismo alternativo también se desarrolla principalmente en áreas naturales protegidas donde los terrenos son propiedad del gobierno que las administra bajo la categoría de bienes públicos. Sin embargo en México permanecen, en su mayor extensión, sujetos a la propiedad privada, ejidal o comunal (Arellano et al., 2008).

\section{IMPACTOS DEL TURISMO EN LAS AVES}

A la fecha se ha sugerido que las actividades turísticas con base en la naturaleza, tales como caminatas, campismo, y el uso de bicicletas, motos de montaña, tienen un efecto negativo en la fauna, incluyendo las aves. El turismo de naturaleza parece afectar directa o indirectamente a gran cantidad de especies de fauna silvestre, incluidas las aves (Gill, 2007). Algunos estudios han determinado que los efectos negativos de estas actividades son generados inicialmente por la alteración o disturbio de sus ambientes (Remacha et al., 2016). Sin embargo también se ha sugerido que la presencia humana y el ruido generado por estas actividades pueden también afectar directamente aspectos fisiológicos y conductuales de la fauna (Steven, 2011; Lindsay et al., 2008).

Aun cuando varios estudios han encontrado resultados sobre los impactos del turismo de naturaleza sobre las aves (Tabla 2), aun se requieren análisis adicionales y rigurosos que permitan determinar estos efectos, ya sea, con análisis estadísticos más poderosos y diseños experimentales rigurosos, o enfocándose hacia ambientes con gran diversidad. Por ejemplo, la mayoría de la investigación de los impactos en las aves por el turismo se ha efectuado en países donde existe poca riqueza de especies, mientras que sitios considerados como de alta riqueza de especies (hot spots) cercanos al trópico no han sido considerados (Steven, 2011). Por ello, es necesario que cada país cuente con evaluaciones propias en sus entornos y ambientes que alojan desarrollos turísticos. Los regímenes de manejo y los patrones de comportamiento humano también pueden diferir de un país a otro, pero estas diferencias son menos críticas que las variaciones entre ecosistemas (Buckley, 2005). 
Tabla 2. Distintos efectos fisiológicos y en el comportamiento de las aves (modificado de Steven 2011).

\begin{tabular}{l|l} 
Efecto fisiológico & Efecto en el comportamiento \\
\hline $\begin{array}{l}\text { Cambio en la temperatura (Müllner } \text { et } \\
\text { al., 2004; Thiel } \text { et al., 2008) }\end{array}$ & Forrajeo (Liddle, 1997; Kirika, 2008) \\
\hline $\begin{array}{l}\text { Arritmias cardiacas (Regel \& Putz, } \\
1997 ; \text { Buckley, 2004) }\end{array}$ & Mayor vigilancia (Lindsay et al., 2008) \\
\hline $\begin{array}{l}\text { Stress en la secreción de hormonas } \\
\text { (Müllner et al.2004; Thiel et al 2008) }\end{array}$ & Evasión (Banks \& Bryant, 2007) \\
\hline & $\begin{array}{l}\text { Éxito reproductivo (Anderson, 2015; Remacha } \\
\text { et al., 2016) }\end{array}$ \\
\hline & $\begin{array}{l}\text { Disminución en la cantidad de nidos, huevos y } \\
\text { polluelos (Cardoni et al., 2008) }\end{array}$ \\
\hline & Menor eclosión de huevos (Buckley, 2004) \\
\hline
\end{tabular}

\subsection{Aspectos fisiológicos y del comportamiento}

Las aves silvestres tienen una alimentación muy especializada y técnicas para encontrar y capturar presas, y han desarrollado un alto grado de especificidad en las imágenes de búsqueda para la detección de sus presas (Pearson, 1977; Remsen \& Parker, 1984), todos estos aspectos moldean sus patrones diarios de actividad. En este sentido, se ha reportado que algunos efectos a nivel fisiológico de las aves, se reflejan en la alteración de los niveles hormonales que provocan stress, influyendo en su temperatura corporal y el ritmo cardiaco (Müllner et al., 2004; Thiel et al., 2008). Todo esto en consecuencia, afecta los patrones de conducta de las aves sometidas a estas actividades. Por ejemplo, algunos estudios han demostrado que la presencia humana afecta los patrones de alimentación de las aves frugívoras (Kirika et al., 2008). Asi mismo, se ha mostrado que las aves tienden a ser más crípticas y evasivas cuando el número de visitantes es mayor en las zonas turísticas (Remacha et al., 2011). Algunas poblaciones de aves incluso pueden aumentar su población al no ser depredados por la presencia humana, lo cual afecta aspectos demográficos de estas especies (Lindsay et al., 2008).

Desde una perspectiva de manejo sustentable, es deseable que las estrategias y acciones empleadas en las zonas donde el turismo de naturaleza se lleva a cabo estén encaminadas en encontrar un equilibrio entre el tamaño de los grupos de visitantes y el número total de visitantes en un momento dado dentro de un área natural (Remacha 2011). En este sentido, se ha sugerido que aquellas áreas de recreación donde la dispersión de las personas no está 
controlada, particularmente en las zonas de amortiguamiento, hay un aumento en las aves de la percepción de riesgo de ser depredadas (Remacha et al., 2016).

Por último, aunque el turismo alternativo también se ha vuelto una opción para los aficionados de las aves, sobre todo aquellos que disfrutan de su observación y registro, es paradójico que se desconozcan los efectos de estas actividades sobre el grupo. Las caminatas para buscarlas, los acercamientos a sus áreas de nidificación o alimentación, la elevada permanencia en sitios específicos para fotografiarlas, son actividades que requieren un análisis para determinarlas como posible impactos (Sekercioglu, 2002), y que mientras tanto permanecen como importantes sesgos en esta asombrosa línea de investigación.

\section{CONCLUSIONES}

Existe una fuerte corriente de investigaciones y acercamientos sobre el efecto del turismo de naturaleza en la fauna silvestre, particularmente las aves. Hay que considerar que este tipo de turismo es relativamente nuevo y para el estudio de las aves y el turismo ejerce una presión a la vida silvestre que se ha ido estudiando muy poco debido a la velocidad de cambio en los ecosistemas más frágiles, y que buscan la sostenibilidad de los ecosistemas, la sociedad y las especies. Sin embargo, la falta de planeación sobre las acciones que implica esta actividad puede ejercer una presión en el ambiente que llegue a tener afectaciones a largo plazo, como la extinción de especies o su desplazamiento permanente. Si bien esta pequeña revisión hace un esfuerzo por indagar en la información descrita para la comprensión de los efectos que pudieran tener las aves por las actividades turísticas, no es suficiente y se recomienda consultar más fuentes para cada impacto descrito en la misma.

\section{CONFLICTO DE INTERESES}

Los autores declaran que no existe conflicto de intereses. 


\section{REFERENCIAS}

Arellano G. A., Fraga J. \& Robles de Benito R. 2008. Descentralización y Manejo Ambiental Gobernanza Costera en México. CINVESTAV IPN. México. 101-116 pp.

Bello I. 2014. Diagnóstico integrado de la actividad turística en la zona costera mexicana. Tesis. UNAM. 156p.

Banks P. \& Bryant, J., 2007. Four-legged friend or foe? Dog walking displaces native birds from natural areas. Biology Letters. 3: 611613.

Buckley R. 2004. Impacts of ecotourism on birds. Environmental Impacts of Ecotourism. CAB International, Cambridge. pp. 187e209.

Buckley R. 2005. Recreation ecology research effort: an international comparison. Tourism Recreation Research. 30(1): 99-101.

García de Fuentes A., Jouault S. \& Romero D. 2015. Atlas de Turismo alternativo en la Península de Yucatán. CINVESTAV Unidad Mérida.170pp.

Cardoni D.A., Favero M. \& Isacch J.P. 2008. Recreational activities affecting the habitat use by birds in Pampa's wetlands, Argentina: implications for waterbird conservation. Biological Conservation. 141, 797806.

Ibáñez Reyna. 2007. Turismo alternativo, gestión y desarrollo local: El caso de Cabo Pulmo, B.C.S. Tesis de Maestría. UABCS. La Paz, México.

Kirika J., Farwig N. \& Bohning-Gaese K. 2008. Effects of local disturbance of tropical forests on frugivores and seed removal of a small-seeded afrotropical tree. Conservation Biology. 22: 318328.

Liddle M. 1997. Recreation Ecology. Chapman and Hall. London.

Liley D. \& Sutherland W. 2007. Predicting the population consequences of human disturbance for ringed plovers Charadrius hiaticula: a game theory approach. Ibis. 149: 8294.

Lindsay K., Craig J. \& Low M., 2008. Tourism and conservation: the effects of track proximity on avian reproductive success and nest selection in an open sanctuary. Tourism Management. 29: $730 \mathrm{e} 739$.

Müllner A., Linsenmair K.E. \& Wikelski M. 2004. Exposure to ecotourism reduces survival and affects stress responses in hoatzin chicks (Opisthocomus hoazin). Biological Conservation 118: 549558. 
OMT. 2005. Guía de indicadores de desarrollo sostenible para destinos turísticos. 28 pp.

Regel J. \& Pütz K.1997. Effect of human disturbance on body temperature and energy expenditure in penguins. Polar Biology 18, $246 \mathrm{e} 253$.

Remacha C., Pérez-Tris J. \& Delgado JA. 2011. Reducing visitors group size increases the number of birds during educational. Journal of Environmental Management. 92: 15641568 .

Remacha C., Delgado JA., \& Mateja Bulaic, Peréz-Tris J. 2016. Human disturbance during early life impairs nestling growth in birds inhabiting a nature recreation area. PLoS ONE 11(11): e0166748. DOI:10.1371

Sekercioglu C. 2002. Impacts of bird watching on human and avian communities. Environmental Conservation. 29: 282_289.

Steven R, Pickering C. \& Castley JG. 2011. A review of the impacts of nature based recreation on birds. J Environ Manage 92: 2287-2294.

Steven R., Guy Castley J. \& Buckley R. 2013. Tourism Revenue as a Conservation Tool for Threatened Birds in Protected Areas. ICER. Australia. 8p.

Thiel D., Jenni-Eiermann S., Braunisch V., Palme R. \& Jenni L. 2008. Ski tourism affects habitat use and evokes a physiological stress response in capercaillie Tetrao urogallus: a new methodological approach. Journal of Applied Ecology. 45, 845e853.

Thiel D., Jenni-Eiermann S., Braunisch V., Palme R. \& Jenni L. 2008. Ski tourism affects habitat use and evokes a physiological stress response in capercaillie Tetrao urogallus: A new methodological approach. J. Appl. Ecol. 45:845-853. 